\title{
Clinical and Pathological Parameters Predicting Extracapsular Disease in Patients Undergoing a Radical Prostatectomy for Clinically Localized Prostate Cancer
}

\section{Sfoungaristos S., Perimenis P.}

Department of Urology, University Hospital of Patras, Patras, Greece

Received April 14, 2011; Accepted January 16, 2012.

Key words: Extracapsular disease - Nerve sparing - Predictors - Prostate cancer Radical prostatectomy

\begin{abstract}
To determine the epidemiological, clinical and pathological factors that can potentially predict extracapsular extension of prostate cancer in patients undergoing radical prostatectomy for clinically localized disease. We retrospectively analyzed the medical records of patients who undergone radical prostatectomy from January 2001 until November 2010. Patients age, prostate volume, PSA, PSA density, percent of cancer in prostate biopsy material, presence of cancer in biopsy cores of the right lobe or the left lobe or both lobes, Gleason summary, $1^{\text {st }}$ Gleason pattern and $2^{\text {nd }}$ Gleason pattern were analyzed for their predictive ability. From 187 studied patients, 44 of them (23.5\%) had extracapsular disease. Multivariate analyses revealed that smaller prostate volumes and the presence of malignancy in both lobes after prostate biopsy were significant predictors for non-organ confined disease in the total population and in patients with Gleason score $\geq 7$. Presence of malignancy in both lobes was the only significant predictive factor in patients with PSA $\leq 10$ and in those with Gleason score $\leq 6$. Prostate volume and positive cores for malignancy from both lobes after prostate biopsy are preoperative data that can be used for prediction of extracapsular disease. This information can be valuable in cases a nerve sparing radical prostatectomy is planned.
\end{abstract}

Mailing Address: Stavros Sfoungaristos, MD., Department of Urology, University Hospital of Patras, Building A, $4^{\text {th }}$ Floor, Rion, 26500 Patras, Greece; Phone: +302 610999 367; Fax: +302 610993 981; e-mail: sfoungaristosst@gmail.com 


\section{Introduction}

The introduction of prostate-specific antigen (PSA) and other circulating biomarkers in addition to transrectal ultrasound has revolutionized prostate cancer (PCa) screening. These factors have resulted in early detection and increased incidence of the disease (Bensalah et al., 2008). Radical prostatectomy is the gold standard for the treatment of clinical localized PCa. However, histopathological examination of the surgical specimen not rarely reveals, that the disease extends outside of the prostatic capsule border. Extracapsular extension is classified as T3a disease, based on 2009 TNM (tumor, node and metastasis) classification and represents a negative pathological factor. These patients have an increased risk for local or systemic disease recurrence and biochemical failure (Lu-Yao et al., 1996). For this reason, preoperative estimation of each patient specific risk for extracapsular disease (ECD), based on epidemiological, clinical and pathological data, would be helpful for the selection of candidates for more aggressive surgical modalities, like neurovascular bundle wide excision, during radical prostatectomy (Smith et al., 1996).

The aim of this study was to analyze several preoperative factors and evaluate their predictive potential for ECD in patients undergoing radical prostatectomy for clinically localized PCa.

\section{Material and Methods}

We performed a retrospective analysis of the medical records from patients who undergone a radical prostatectomy with the diagnosis of organ-confined PCa from January of 2001 until November of 2010. The study protocol had been approved by Ethics Committee of our institution and it conformed to the provisions of the Declaration of Helsinki in 1995 (as revised in Edinburgh 2000). Exclusion criteria from the study was any preoperative therapy for PCa (hormone therapy, radiation therapy) and patients that found to have seminal vesicle invasion (stage T3b) or invasion of adjacent structures like bladder neck and external sphincter (stage T4). Patients diagnosed after transurethral resection of the prostate were excluded. Patients with incomplete medical records were excluded, as well. The analysis comprised preoperative $1^{\text {st }}$ and $2^{\text {nd }}$ Gleason pattern and Gleason summary, age, preoperative value of PSA, prostate volume, PSA density, the presence of positive cores for malignancy after prostate biopsy in right $(R+)$ or left $(L+)$ or both $(R L+)$ prostatic lobes and percentage of cancer in biopsy material (\% CM).

Preoperative PSA was measured before digital rectal examination, transrectal ultrasound or biopsy. In all patients cancer suspicion, because of PSA elevation, abnormal digital rectal examination or hypoechoic and hypervascular areas in Doppler transrectal ultrasound, was confirmed by transrectal ultrasound biopsy and positive for malignancy histological examination of the obtained cores.

An open retropubic or laparoscopic extraperitoneal radical prostatectomy was performed in all patients by 4 experienced surgeons. The surgical specimen was 
sent for pathological examination and a histological report concerning the prostate dimensions, GS and $1^{\text {st }}$ and $2^{\text {nd }}$ pattern and pathological stage was obtained. As stage T3a was considered disease extension outside of the prostate capsule. When the disease had penetrated the prostate capsule but was not extended outside this, the disease was staged as T2c.

Prostate volume was calculated according to the information of the maximum transverse diameter (D1), the maximum anteroposterior diameter (D2) and the maximum longitudinal diameter (D3) and by using the formula D $1 \times D 2 \times D 3 \times \pi / 6$ based on the prostate ellipse dimension theory. PSA density was calculated by dividing the preoperative PSA value and prostate volume. Even thought prostate volume was calculated postoperatively according to the prostate dimensions given by the pathologists report, there is a great positive correlation between preoperative (during transrectal ultrasound) and postoperative calculation of prostate volume (Wolff et al., 1995).

The study design had 5 objectives. The first one was to analyze the above mentioned parameters as predictors for ECD in the total population of patients. Next, we analyze the same factors and their predictive ability for ECD in patients with PSA $\leq 10 \mathrm{ng} / \mathrm{ml}$ (subgroup 1), in patients with PSA >10 ng/ml (subgroup 2), in patients with preoperative Gleason score $\leq 6$ (subgroup 3 ) and in patients with preoperative Gleason score $\geq 7$ (subgroup 4).

\section{Statistical analysis}

All analyses were performed by using SPSS version 17 (SPSS Inc., Chicago, IL, USA). The descriptive statistics are presented as the mean \pm standard deviation (SD) and interquartile range (IQR) for continuous variables and as the absolute and percent frequency for categorical variables.

The normality condition of the numerical variables was studied by means of the Kolmogorov-Smirnov test. None of them had normal distribution. For this reason, the Mann-Whitney test was used to compare means between numerical groups. The chi-square $\chi^{2}$ test was used for categorical variables. A univariate analysis was performed to identify the predictive significance of age, preoperative PSA, prostate volume, PSA density, preoperative Gleason score, $\mathrm{R}+, \mathrm{L}+, \mathrm{RL}+$ and \% CM in prediction of ECD. A multivariate analysis was performed then for the variables identified as statistically important in univariate analysis, using logistic regression. The same statistical concatenation was used for subgroups data analysis, as well. By using receiver operating curve (ROC curve), the area under the curve (AUC) was derived. The optimal cutoff value and sensitivity and specificity for quantitative variables were estimated by using ROC curve, as well. In case of quantitative variables, cross tabulation was used for calculation of sensitivity [number of true positive/ (number of true positive + number of false negative)] and specificity [number of true negative/(number of true negative + number of false positive)]. Positive [true positive/(true positive + false positive)] and negative predictive value [true negative/ 
(true negative + false negative)] were estimated as well. All tests were 2-tailed with $\alpha$ of $p$-value of less than 0.05 to be considered as statistically significant.

\section{Results}

A total of 187 patients were reviewed finally. Patients' age were ranged from $46-78$ years $(66.8 \pm 6.3,9)$. Preoperative PSA value ranged from $3.3-41.8 \mathrm{ng} / \mathrm{ml}$ $(9.9 \pm 5.8,5.2)$, PSA density from $0.01-1.85(0.26 \pm 0.24,0.16)$ and prostate volume from $7-140 \mathrm{ml}(45.34 \pm 22.01,28)$. After a median number of 12.00 cores $(12.79 \pm 6.70,10)$ obtained during prostate biopsy procedures, 39 patients $(20.9 \%)$ had $\mathrm{R}+, 44$ patients (23.5\%) had $\mathrm{L}+$ and 104 patients (55.6\%) had RL+. The \% CM was ranged from $0.04-90 \%(26.75 \pm 23.43,31)$. Preoperative Gleason score $\leq 6$ was found in 108 patients (57.8\%) and 79 patients (42.2\%) had Gleason score $\geq 7$. The pathological examination of the surgical specimens revealed that 44 patients (23.5\%) had ECD. The characteristics of the patients with and without ECD are seen in Table 1. A lymphadenectomy procedure was performed in 124 patients (66.3\%) and positive lymph node metastasis was observed in 13 of them (10.5\%).

In multivariate analysis of the variables found to be statistically significant in univariate analysis (prostate volume, PSA density, $\mathrm{R}+, \mathrm{RL}+, \% \mathrm{CM}$, Gleason score and $1^{\text {st }}$ pattern of Gleason score $)$, we found that only prostate volume $(p=0.028)$ and $R L+(p=0.034)$ were significant for prediction of ECD (Table 2).

By using ROC curve, the AUC for prostate volume and LR+ were $0.666(p=0.001$, $95 \% \mathrm{Cl} 0.578-0.753)$ and $0.686(p<0.001,95 \% \mathrm{Cl} 0.602-0.770)$, respectively. Sensitivity and specificity of RL+ to predict ECD were $84.1 \%$ and $53.1 \%$, respectively. ROC curve analysis showed that an optimal cut off value for prostate volume is $\leq 40 \mathrm{ml}$ which is correlated with ECD with $77.3 \%$ sensitivity and $52.4 \%$ specificity.

\section{Subgroup 1 analysis}

One hundred and thirty one patients (70.05\%) had PSA $\leq 10 \mathrm{ng} / \mathrm{ml}$ and 25 of them (19.1\%) had ECD. In univariate analysis of preoperative variables for prediction of ECD in this group of patients, we found that prostate volume $(p=0.001)$, PSA density $(p=0.003), R L+(p<0.001), R+(p=0.025), L+(p=0.042), \%$ CM $(p=0.008)$, Gleason score $(p=0.026)$ and $2^{\text {nd }}$ Gleason pattern $(p=0.031)$ were statistically significant, while age $(p=0.404)$, PSA $(p=0.731)$ and $1^{\text {st }}$ Gleason pattern $(p=0.098)$ were not. The multivariate analysis is seen in Table 3 . The only factor found to be significant was $R L+(p=0.005)$. AUC was $0.737(p<0.001,95 \% \mathrm{Cl} 0.639-0.836)$. $\mathrm{RL}+$ can predict ECD in patients with PSA $\leq 10 \mathrm{ng} / \mathrm{ml}$ with $88.0 \%$ sensitivity and $59.4 \%$ specificity.

\section{Subgroup 2 analysis}

Fifty six patients (29.95\%) had PSA $>10 \mathrm{ng} / \mathrm{ml}$ and 19 of them $(33.9 \%)$ had ECD. Interestingly, as the results of the univariate analysis shown, none of the 
Table 1 - Patients' characteristics

\begin{tabular}{|c|c|c|c|}
\hline & \multicolumn{2}{|c|}{ Pathological stage } & \multirow[b]{2}{*}{$P$} \\
\hline & organ confined & extracapsular & \\
\hline \multicolumn{4}{|l|}{ Age (years) } \\
\hline mean $\pm S D(I Q R)$ & $66.64 \pm 6.36(9)$ & $67.30 \pm 6.07(8)$ & $0.640^{x}$ \\
\hline \multicolumn{4}{|l|}{ Prostate volume $(\mathrm{ml})$} \\
\hline mean $\pm \mathrm{SD}(\mathrm{IQR})$ & $48.01 \pm 22.62(30)$ & $36.68 \pm 17.50(15)$ & $0.001^{x *}$ \\
\hline \multicolumn{4}{|l|}{ PSA (ng/ml) } \\
\hline mean $\pm S D(I Q R)$ & $9.52 \pm 5.87(4.28)$ & $10.98 \pm 5.49(8.00)$ & $0.046^{\times *}$ \\
\hline \multicolumn{4}{|l|}{ PSA density $\left(\mathrm{ng} / \mathrm{ml}^{2}\right)$} \\
\hline mean $\pm \mathrm{SD}(\mathrm{IQR})$ & $0.25 \pm 0.23(0.15)$ & $0.35 \pm 0.27(0.12)$ & $<0.001^{1 *}$ \\
\hline \multicolumn{3}{|l|}{$\mathrm{RL}+, \mathrm{n}(\%)$} & $<0.001^{x \times *}$ \\
\hline yes & $67(64.4)$ & $37(35.6)$ & \\
\hline no & $76(91.6)$ & $7(8.4)$ & \\
\hline \multicolumn{3}{|l|}{$\mathrm{R}+, \mathrm{n}(\%)$} & $0.001^{x \times *}$ \\
\hline yes & $38(97.4)$ & $1(2.6)$ & \\
\hline no & $105(70.9)$ & $43(29.1)$ & \\
\hline \multicolumn{3}{|l|}{$L+, n(\%)$} & $0.077^{x x}$ \\
\hline yes & $38(86.4)$ & $6(13.6)$ & \\
\hline no & $105(73.4)$ & $38(26.6)$ & \\
\hline \multicolumn{3}{|l|}{$\% C M$} & $0.001^{\times *}$ \\
\hline mean $\pm S D(I Q R)$ & $23.50 \pm 1.30(29)$ & $37.32 \pm 25.34(44)$ & \\
\hline \multicolumn{3}{|l|}{ GS, n (\%) } & $0.159^{x x}$ \\
\hline 2 & $2(100.0)$ & $0(0.0)$ & \\
\hline 3 & $6(100.0)$ & $0(0.0)$ & \\
\hline 4 & $8(100.0)$ & $0(0.0)$ & \\
\hline 5 & $20(90.9)$ & $2(9.1)$ & \\
\hline 6 & $50(71.4)$ & $20(28.6)$ & \\
\hline 7 & $43(69.4)$ & $19(30.6)$ & \\
\hline 8 & $10(83.3)$ & $2(16.7)$ & \\
\hline 9 & $4(80.0)$ & $1(20.0)$ & \\
\hline \multicolumn{3}{|l|}{$1^{\text {st }}$ pattern, n (\%) } & $0.028^{x \times *}$ \\
\hline 1 & $2(100.0)$ & $0(0.0)$ & \\
\hline 2 & $25(96.2)$ & $1(3.8)$ & \\
\hline 3 & $87(75.0)$ & $29(25.0)$ & \\
\hline 4 & $25(64.1)$ & 14 (35.9) & \\
\hline 5 & $4(100.0)$ & $0(0.0)$ & \\
\hline \multicolumn{3}{|l|}{$2^{\text {nd }}$ pattern, n (\%) } & $0.096^{x x}$ \\
\hline 1 & $8(100.0)$ & $0(0.0)$ & \\
\hline 2 & $18(94.7)$ & $1(5.3)$ & \\
\hline 3 & 79 (71.2) & $32(28.8)$ & \\
\hline 4 & $33(76.7)$ & $10(23.3)$ & \\
\hline 5 & $5(83.3)$ & $1(16.7)$ & \\
\hline
\end{tabular}


Table 2 - Univariate and multivariate analysis of epidemiologic, clinical and pathological factors for extracapsular disease prediction in the total population of patients

\begin{tabular}{|c|c|c|c|c|}
\hline & \multicolumn{4}{|c|}{$95 \% \mathrm{Cl}$} \\
\hline & Significance & Odds ratio & Lower & Upper \\
\hline \multicolumn{5}{|c|}{ Univariate analysis } \\
\hline Age & 0.546 & 1.017 & 0.963 & 1.075 \\
\hline PV & $0.004 *$ & 0.970 & 0.950 & 0.990 \\
\hline PSA & 0.155 & 1.039 & 0.986 & 1.096 \\
\hline PSAD & $0.026^{*}$ & 4.640 & 1.203 & 17.895 \\
\hline $\mathrm{RL}+$ & $0.000 *$ & 5.996 & 2.507 & 14.341 \\
\hline $\mathrm{R}+$ & $0.008 *$ & 0.064 & 0.009 & 0.483 \\
\hline$L+$ & 0.083 & 0.436 & 0.171 & 1.114 \\
\hline$\% \mathrm{CM}$ & $0.001 *$ & 1.024 & 1.010 & 1.038 \\
\hline GS & $0.036 *$ & 1.388 & 1.022 & 1.885 \\
\hline $1^{\text {st }}$ pattern & $0.025^{*}$ & 1.793 & 1.075 & 2.992 \\
\hline $2^{\text {nd }}$ pattern & 0.170 & 1.366 & 0.875 & 2.132 \\
\hline \multicolumn{5}{|c|}{ Multivariate analysis } \\
\hline PV & $0.028 *$ & 0.971 & 0.946 & 0.997 \\
\hline PSAD & 0.991 & 1.009 & 0.210 & 4.851 \\
\hline $\mathrm{RL}+$ & $0.034 *$ & 3.057 & 1.090 & 8.575 \\
\hline $\mathrm{R}+$ & 0.103 & 0.161 & 0.018 & 1.442 \\
\hline$\% \mathrm{CM}$ & 0.416 & 1.007 & 0.990 & 1.024 \\
\hline GS & 0.875 & 1.045 & 0.604 & 1.806 \\
\hline $1^{\text {st }}$ pattern & 0.390 & 1.528 & 0.582 & 4.014 \\
\hline
\end{tabular}

*statistically significant; PV - prostate volume; PSA - prostate specific antigen; PSAD - prostate specific antigen density; $\mathrm{R}+-$ presence of cancer in biopsy cores of right lobe; $\mathrm{L}+-$ presence of cancer in biopsy cores of left lobe; RL+ presence of cancer in biopsy cores of both lobes; \% CM - percent of cancer in biopsy material; GS - Gleason score; $\mathrm{Cl}$ - confidence interval

preoperative factors was significant in prediction of T3a disease and consequently no multivariate analysis was performed.

\section{Subgroup 3 analysis}

One hundred and eight patients (57.75\%) from the study population had a biopsy Gleason score $\leq 6$. Twenty two of them $(20.4 \%)$ had ECD. Univariate analysis in this subgroup revealed that $R L+(p=0.008), 1^{\text {st }}$ Gleason pattern $(p=0.034), 2^{\text {nd }}$ Gleason pattern $(p=0.040)$ and Gleason score $(p=0.023)$ were significant for ECD prediction, while age $(p=0.773)$, prostate volume $(p=0.094)$, PSA $(p=0.468)$, PSA density $(p=0.105), R+(p=0.998), L+(p=0.444)$ and \% CM $(p=0.125)$ were not. In multivariate analysis (Table 3$)$, only $R L+(p=0.018)$ was found to be significant in prediction of ECD. AUC was $0.670(p=0.014,95 \% \mathrm{Cl} 0.554-0.786)$. Sensitivity and specificity of RL+ to predict ECD in patients with Gleason score $\leq 6$ was $86.4 \%$ and $47.7 \%$, respectively. 
Table 3 - Multivariate analyses in subgroups 1, 3 and 4

\begin{tabular}{|c|c|c|c|c|}
\hline & \multicolumn{4}{|c|}{$95 \% \mathrm{Cl}$} \\
\hline & Significance & Odds ratio & Lower & Upper \\
\hline \multicolumn{5}{|c|}{ Multivariate analysis in patients with PSA $\leq 10$} \\
\hline PV & 0.215 & 0.961 & 0.903 & 1.023 \\
\hline PSAD & 0.138 & 796.068 & 0.116 & 5454287.548 \\
\hline $\mathrm{RL}+$ & $0.005^{*}$ & 15.208 & 2.264 & 102.145 \\
\hline $\mathrm{R}+$ & 0.344 & 0.208 & 0.008 & 5.372 \\
\hline$\% C M$ & 0.952 & 1.001 & 0.977 & 1.025 \\
\hline GS & 0.072 & 2.865 & 0.909 & 9.027 \\
\hline $2^{\text {nd }}$ pattern & 0.579 & 0.631 & 0.124 & 3.212 \\
\hline \multicolumn{5}{|c|}{ Multivariate analysis in patients with GS $\leq 6$} \\
\hline $\mathrm{RL}+$ & $0.018 *$ & 4.927 & 1.315 & 18.459 \\
\hline GS & 0.172 & 3.739 & 0.564 & 24.764 \\
\hline $1^{\text {st }}$ pattern & 0.838 & 1.356 & 0.074 & 24.884 \\
\hline \multicolumn{5}{|c|}{ Multivariate analysis in patients with GS $\geq 7$} \\
\hline PV & $0.029 *$ & 0.957 & 0.920 & 0.995 \\
\hline $\mathrm{RL}+$ & $0.015^{*}$ & 5.616 & 1.402 & 22.488 \\
\hline$\% C M$ & 0.425 & 1.010 & 0.986 & 1.034 \\
\hline
\end{tabular}

*statistically significant; other abbreviations like Table 2

Table 4 - Predictive accuracy of the variables found significant in multivariate analyses

\begin{tabular}{lccccc}
\hline & Predictive factor & Sensitivity & Specificity & PPV & $\mathrm{npv}$ \\
\hline Total population & $\mathrm{PV} \leq 40 \mathrm{ml}$ & $77.3 \%$ & $52.4 \%$ & $33.3 \%$ & $88.2 \%$ \\
& $\mathrm{RL}+$ & $84.1 \%$ & $53.1 \%$ & $35.6 \%$ & $91.6 \%$ \\
\hline Subgroup 1 & $\mathrm{RL}+$ & $88.0 \%$ & $59.4 \%$ & $33.8 \%$ & $95.5 \%$ \\
\hline Subgroup 3 & $\mathrm{RL}+$ & $86.4 \%$ & $47.7 \%$ & $29.7 \%$ & $93.2 \%$ \\
\hline Subgroup 4 & $\mathrm{PV} \leq 40 \mathrm{ml}$ & $81.8 \%$ & $49.1 \%$ & $38.3 \%$ & $87.5 \%$ \\
& $\mathrm{RL}+$ & $81.8 \%$ & $61.4 \%$ & $45.0 \%$ & $89.7 \%$ \\
\hline
\end{tabular}

Ppv - positive predictive value; npv - negative predictive value; other abbreviations like Table 2

Subgroup 4 analysis

In this subgroup, 79 patients (42.25\%) were included and 22 of them (27.8\%) had ECD. Prostate volume $(p=0.013), R L+(p=0.001)$ and $\% C M(p=0.008)$ were significant in univariate analysis. On the other hand, patients age $(p=0.705)$, PSA $(p=0.294)$, PSA density $(p=0.131), R+(p=0.059), L+(p=0.071)$, Gleason score $(p=0.359), 1^{\text {st }}$ Gleason pattern $(p=0.696)$ and $2^{\text {nd }}$ Gleason pattern $(p=0.213)$ were not statistically significant. In the multivariate analysis (Table 3 ) we found that $R L+(p=0.015)$ and prostate volume $(p=0.029)$ are the preoperative factors that can predict ECD in patients with preoperative Gleason score $\geq 7$. AUC was 0.716 
$(p=0.003,95 \% \mathrm{Cl} 0.594-0.839)$ for $\mathrm{RL}+$ and $0.692(p=0.009,95 \% \mathrm{Cl} 0.569-0.815)$ for prostate volume. An optimal cutoff for prostate volume was a value $\leq 40 \mathrm{ml}$ with $81.8 \%$ sensitivity and $31.6 \%$ specificity. The sensitivity and specificity of RL+ to predict ECD was $81.8 \%$ and $49.1 \%$, respectively (Table 4 ).

\section{Discussion}

In the era of PSA, the incidence and detection of PCa has increased and more patients with insignificant cancers are operated. Given the fact that a significant number of young patients are undergoing radical prostatectomy, a specific attention has been given in the postoperative functional results, like potency and continence. Radical retropubic prostatectomy with neurovascular bundle preservation was firstly described by Walsh and since then this surgical procedure is widely used worldwide (Walsh et al., 1983). However, this technique can lead to an increased incidence of positive surgical margin and incomplete excision of advanced disease like in cases of PCa with extracapsular extension. Since the incidence of early biochemical failure and local or distant recurrence is higher in patients with ECD and furthermore, less aggressive techniques, like nerve-sparing radical prostatectomy, can increase the number of positive surgical margins and incomplete tumor excision in patients with pathological T3a disease, preoperative factors that predict the tumor extend are important. Patients that are suspected for ECD may benefit, by a cancer control standpoint, from a wide neurovascular bundle excision.

Several variables have been proposed as predictors for ECD. Preoperative PSA found to be a significant predictor for tumor extend (Partin et al., 1993; Cookson et al., 1997; Ogawa et al., 1998). The incidence of ECD had a linear relationship with PSA increases, as it was confirmed in a study of patients with clinically localized disease (Partin et al., 1993). In another study of 107 patients, it was reported that PSA level was the strongest predictor for tumor stage and patients with preoperative values $<7 \mathrm{ng} / \mathrm{ml}$ had significantly higher rates of organ confined disease, while patients with PSA $>20 \mathrm{ng} / \mathrm{ml}$ were at higher risk for cancer spread outside prostatic capsule (Cookson et al., 1997). These results suggest that efforts directed toward identifying cancers in patients with early PSA elevations may result in improved rates of organ confined disease. Another study has reported that preoperative PSA with a cutoff value of $17 \mathrm{ng} / \mathrm{ml}$ can predict organ confined disease with $90 \%$ sensitivity and $70 \%$ specificity (Ogawa et al., 1998). Similar results were found by another 2 studies reported that PSA is one of the most significant variables for ECD (Ravery et al., 2000; Ou et al., 2002). However, results from our case series have demonstrated that PSA was not a significant predictor for ECD and this was confirmed by multivariate analyses in the whole amount of studied patients and in patients of the 4 subgroups.

High Gleason score is correlated with non-organ confined disease (Wills et al., 1998; Ou et al., 2002; Ozgür et al., 2004) and patients with poorly differentiated tumors have a higher incidence of extracapsular extension and positive margins (Bigg et al., 1990). Furthermore, in patients with low and moderate grade tumors, 
the neurovascular bundle can be safely preserved on the side without evidence of cancer having obtained at least in 3 biopsy cores (Sanwick et al., 1998). This was not the case of our study in which Gleason score is not predicting the presence of ECD in none of the analyses performed. Furthermore, we evaluated the role of $1^{\text {st }}$ and $2^{\text {nd }}$ Gleason patterns. Although $1^{\text {st }}$ pattern was statistically significant predictor in univariate analysis in the group of the whole population $(p=0.025)$ and in subgroup of patients with Gleason score $\leq 6(p=0.034)$, this was not confirmed in the multivariate analyses with $p$ values to be 0.390 and 0.838 , respectively.

PSA density $\geq 0.35$ has been found to be predictive for ECD in a recent study of 55 patients underwent radical prostatectomy for incidentally found PCa (Ou et al., 2002). In our study, even though the analyzed patients were not only T1c staged, PSA density could not predict cancer extracapsular extension.

Most of the studies published in the literature are analyzing the impact of prostate biopsy cores histological examination results in prediction of ECD. Mainly, the factors which are considered for analysis are the percentage of cancer in biopsy cores and the number or percentage of cores with cancer. More than $20 \%$ of cancer in biopsy material was highly correlated with ECD (Ou et al., 2002), while it has reported that the length of tissue core invaded by cancer is significant for ECD, as well (Ravery et al., 2000). Two recent nomograms have been proposed for prediction of ECD (Ohori et al., 2004; Satake et al., 2010). In both cases, biopsy results (the percent of positive cores and percent of cancer in the biopsy specimen) added in the standard clinical features most commonly used for prediction (PSA, Gleason score and clinical stage). There was an excellent calibration and a significant increase in prediction and discrimination accuracy of the developed models after internal validation. The number of positive biopsy cores may be useful as pretreatment parameter to identify patients with a higher likelihood for organ confined disease (Ogawa et al., 1998; Wills et al., 1998; Ravery et al., 2000; Mortensen et al., 2009). In our analysis, although \% CM was found to be correlated with ECD in univariate analyses of the whole population $(p=0.001)$ and subgroup $1(p=0.008)$ and $4(p=0.008)$, no statistically importance was noticed in multivariate analyses $(p=0.416, p=0.952$, $\mathrm{p}=0.425$, respectively).

The present study has a number of limitations that should be mentioned. The data collection was made in a retrospective fashion and this is limited the power of the results. A large number of medical records were incomplete and consequently a great number of patients were excluded from the study. Given the fact that the study period was approximately 10 years and surgical experience and concepts have been revolutionized in this time period, the small number of analyzed patients may limiting the significance of the results. Moreover, the procedures have been made by 4 different operators with different surgical experience and philosophy and we believe that inter-operator variability may affect the pathological outcome.

In our study, when we analyzed the predictive accuracy of several preoperative factors in total population of 187 patients, prostate volume $(p=0.028)$ and $R L+$ 
$(p=0.034)$ were the only variables found to be significant in multivariate analysis. Similar results were noticed in subgroup of patients with Gleason score $\geq 7$, but in this case RL+ found to be more significant than prostate volume, with p-values of 0.015 and 0.029 , respectively. Furthermore, $R L+$ was the only significant predictor in patients with Gleason score $\leq 6(p=0.018)$ and in patients with PSA $\leq 10 \mathrm{ng} / \mathrm{ml}$ $(p=0.005)$.

\section{Conclusion}

Prediction of ECD is extremely important both for patients' selection and for prognosis after radical prostatectomy. Based in our knowledge, this is the first study reporting the significance of prostate volume and the presence of positive for cancer cores from both prostatic lobes in prediction of ECD in patients planned for definitive therapy and these results can be a valuable tool in cases a nerve-sparing radical prostatectomy is planned.

\section{References}

Bensalah, K., Lotan, Y., Karam, J. A., Shariat, S. F. (2008) New circulating biomarkers for prostate cancer. Prostate Cancer Prostatic Dis. 11, 112-120.

Bigg, S. W., Kavoussi, L. R., Catalona, W. J. (1990) Role of nerve-sparing radical prostatectomy for clinical stage B2 prostate cancer. J. Urol. 144, 1420-1424.

Cookson, M. S., Fleshner, N. E., Soloway, S. M., Fair, W. R. (1997) Prognostic significance of prostate-specific antigen in stage T1c prostate cancer treated by radical prostatectomy. Urology 49, 887-893.

Lu-Yao, G. L., Potosky, A. L., Albertsen, P. C., Wasson, J. H., Barry, M. J., Wennberg, J. E. (1996) Follow-up prostate cancer treatments after radical prostatectomy: a population-based study. J. Natl. Cancer Inst. 88, 166-173.

Mortensen, M. M., Mortensen, P. S., Borre, M. (2009) Percentage of tumour-positive biopsy cores: an independent predictor of extraprostatic disease. Scand. J. Urol. Nephrol. 43, 109-113.

Ogawa, O., Egawa, S., Arai, Y., Tobisu, K., Yoshida, O., Kato, T. (1998) Preoperative predictors for organ-confined disease in Japanese patients with stage T1c prostate cancer. Int. J. Urol. 5, 454-458.

Ohori, M., Kattan, M. W., Koh, H., Maru, N., Slawin, K. M., Shariat, S., Muramoto, M., Reuter, V. E., Wheeler, T. M., Scardino, P. T. (2004) Predicting the presence and side of extracapsular extension: a nomogram for staging prostate cancer. J. Urol. 171, 1844-1849.

Ou, Y. C., Chen, J. T., Yang, C. R., Cheng, C. L., Ho, H. C., Kao, Y. L., Ko, J. L., Hsieh, Y. S. (2002) Preoperative prediction of extracapsular tumor extension at radical retropubic prostatectomy in Taiwanese patients with T1c prostate cancer. Jpn. J. Clin. Oncol. 32, 172-176.

Ozgür, A., Onol, F. F., Türkeri, L. N. (2004) Important preoperative prognostic factors for extracapsular extension, seminal vesicle invasion and lymph node involvement in cases with radical retropubic prostatectomy. Int. Urol. Nephrol. 36, 369-373.

Partin, A. W., Yoo, J., Carter, H. B., Pearson, J. D., Chan, D. W., Epstein, J. I., Walsh, P. C. (1993) The use of prostate specific antigen, clinical stage and Gleason score to predict pathological stage in men with localized prostate cancer. J. Urol. 150, 110-114.

Ravery, V., Chastang, C., Toublanc, M., Boccon-Gibod, L., Delmas, V., Boccon-Gibod, L. (2000) Percentage of cancer on biopsy cores accurately predicts extracapsular extension and biochemical relapse after radical prostatectomy for T1-T2 prostate cancer. Eur. Urol. 37, 449-455.

Sfoungaristos S.; Perimenis P. 
Sanwick, J. M., Dalkin, B. L., Nagle, R. B. (1998) Accuracy of prostate needle biopsy in predicting extracapsular tumor extension at radical retropubic prostatectomy: application in selecting patients for nerve-sparing surgery. Urology 52, 814-818.

Satake, N., Ohori, M., Yu, C., Kattan, M. W., Ohno, Y., Miyakawa, A., Hatano, T., Tachibana, M. (2010) Development and internal validation of a nomogram predicting extracapsular extension in radical prostatectomy specimens. Int. J. Urol. 17, 267-272.

Smith, R. C., Partin, A. W., Epstein, J. I., Brendler, C. B. (1996) Extended follow-up of the influence of wide excision of the neurovascular bundle(s) on prognosis in men with clinically localized prostate cancer and extensive capsular perforation. J. Urol. 156, 454-457.

Walsh, P. C., Lepor, H., Eggleston, J. C. (1983) Radical prostatectomy with preservation of sexual function: anatomical and pathological considerations. Prostate 4, 473-485.

Wills, M. L., Sauvageot, J., Partin, A. W., Gurganus, R., Epstein, J. I. (1998) Ability of sextant biopsies to predict radical prostatectomy stage. Urology 51, 759-764.

Wolff, J. M., Boeckmann, W., Mattelaer, P., Handt, S., Adam, G., Jakse, G. (1995) Determination of prostate gland volume by transrectal ultrasound: correlation with radical prostatectomy specimens. Eur. Urol.

28, 10-12. 